La Revue

des Droits

de l'Homme

\section{La Revue des droits de l'homme}

Revue du Centre de recherches et d'études sur les droits fondamentaux

16 | 2019

Revue des droits de l'homme - $\mathrm{N}^{\circ} 16$

Les modalités de conquête du logement aux origines de la territorialisation des asentamientos urbains marginaux de Guayaquil (Equateur).

\title{
Mathias Pécot
}

\section{(2) OpenEdition}

\section{Journals}

Édition électronique

URL : http://journals.openedition.org/revdh/6722

DOI : $10.4000 /$ revdh. 6722

ISSN : 2264-119X

Éditeur

Centre de recherches et d'études sur les droits fondamentaux

Référence électronique

Mathias Pécot, «Les modalités de conquête du logement aux origines de la territorialisation des asentamientos urbains marginaux de Guayaquil (Equateur). », La Revue des droits de l'homme [En ligne], 16 | 2019, mis en ligne le 27 juin 2019, consulté le 08 juillet 2020. URL : http:// journals.openedition.org/revdh/6722 ; DOI : https://doi.org/10.4000/revdh.6722

Ce document a été généré automatiquement le 8 juillet 2020.

Tous droits réservés 


\title{
Les modalités de conquête du logement aux origines de la
} territorialisation des asentamientos urbains marginaux de Guayaquil (Equateur).

\author{
Mathias Pécot
}

1 Pendant sociohistorique des mutations qui traversent le monde rural et d'une démographie ponctuée par un accroissement radical des inégalités, le processus d'exil socioéconomique ou environnemental vers les villes s'accentue inexorablement au fil du XXe siècle ${ }^{1}$. Offrande aux totems de la ville et du développement, le tout-désigné monde des taudis apparait volontiers comme le foyer des populations déplacées, diminuées ou vulnérables. Certains auteurs, comme Manuel Castel ou David Harvey, observent à travers le prisme de l'urbanisation marginale le stigmate d'une situation de dépendance $^{2}$. Que ces poches de peuplement soient aujourd'hui situées dans des régions riches ou pauvres, développées ou en transition, les "épistémologies du Sud" renvoient bien, dans ce contexte et sur un plan symbolique, aux périphéries de la métropole moderne.

2 Affinant le survol, ces grands récits de la croissance urbaine marginale des villes du Sud et, plus spécifiquement de l'urbanisation sud-américaine, laissent néanmoins planer d'importantes zones d'ombres. Le caractère dissonant et, souvent même, contradictoire des mises en perspective d'une vie de bidonvilles avec certains vécus du terrain interpelle. L'évocation du trait de marginalité, alors que la formation et la consolidation desdits asentamientos ( slums » ou « bidonvilles ») font localement figure de phénomènes structurels, catalyseurs de majorités sans précédent, paraît troublante. Du point de vue du droit, l'idée commune est celle d'une absence de formes dévalorisée. Mais au vu des modes de normativités locales, le droit apparaît comme un artifice encombrant. 
3 Près de cinquante ans après les travaux Keith Hart dans le cadre de l'Organisation Internationale du Travail ${ }^{3}$, le prisme résilient de l'informel et le renvoi systématique à l'image d'un méandre asséché de l'ordre juridique local afin de caractériser certaines activités, espaces, personnes ou temporalités attachées à la conquête des droits fondamentaux en ville confine, aujourd'hui, au réductionnisme analytique ${ }^{4}$. L'annihilation à la fois discursive et performative de ce qui s'inscrit sur le chemin tout tracé des idéologies du développement ${ }^{5}$, de l'État de droit $^{6}$, du néolibéralisme urbain ${ }^{7}$, des idéaux humanitaires ou religieux ${ }^{8}$ opère comme un miroir déformant ${ }^{9}$. Ces distorsions en matière de perception et de théorisation des faits de marginalité urbaine, faits de juridicité inclus, soulèvent un problème d'ordre axiologique. Le biais informationnel constitue, de même, un véritable obstacle méthodologique lorsqu'il s'agit de penser l'agir juridique et la dynamique de réalisation des droits dans les asentamientos.

4 Face à cet écueil, le présent travail s'engage sur la voie d'un possible dépassement analytique. Ebauchés à la lumière du cas de Guayaquil, et plus spécifiquement d'une longue immersion dans le secteur associatif local du «Monte Sinaï», les propos qui suivent apportent un regard alternatif sur la vie juridique des asentamientos. Premier pôle migratoire de l'Équateur, la zone d'influence métropolitaine de Guayaquil avoisine actuellement les trois millions d'habitants. Il est estimé, à titre indicatif, que la population urbaine du canton a crû d'un facteur d'un à dix entre 1950 et 2010 . Localisées au Nord-Ouest de la ville, les coopératives du secteur Monte Sinaï comptent, suivant les derniers recensements, près de 270000 habitants. Le flux des arrivants n'a jamais cessé au cours des vingt dernières années. Jusqu'à l'intervention récente du gouvernement central, l'affluence était estimée, en moyenne, à soixante familles par semaine. À quelques encablures du centre-ville, du patchwork de zones "régénérées » et d'urbanisations privées, Monte Sinaï fait figure de localité emblématique. Les problématiques sociales et environnementales d'une «vie de taudis » alimentées par l'imaginaire collectif de l'invasion ne sont rien d'autre, ici, que celles d'un quotidien et d'une promesse de lendemain partagée par ses habitants. La territorialisation des asentamientos et la transformation matérielle des conditions d'existence s'effectuent au grand jour et à pleine vitesse. En quelques années, la Entrada de la 8, point d'entrée principale vers la jungle urbaine est déjà méconnaissable. Voies asphaltées, transformateurs électriques et signalisation, poste de police, transports urbains, services publics, commerces et marchés : la rumeur gronde. La conquête de la ville avance.

5 De l'achat d'une promesse de vente à l'obtention d'un titre de propriété ; du raccord artisanal à l'acquisition du sacro-saint compteur électrique ; de l'achat de l'eau potable dutanquero- un camion-citerne assurant le service d'approvisionnement quotidien de l'asentamiento - à la première facture d'eau; de la latrine aux toilettes ; des quatre murs préfabriqués de la casa Hogar de Cristo au ciment ; du botadero - le point de décharge à ciel ouvert des déchets ménagers - à l'inscription de la cuadra - l'unité minimale de planification urbaine - sur une route de collecte publique; du service prépayé de téléphonie mobile au premier cyber-café; des trici-moto aux alimentadores - les navettes assurant une connexion directe au réseau de transport municipal «métrovia»- en passant par la desserte des coopératives de transport urbain; du campement scolaire à l'école publique; du dispensario comme première offre de soin médical assurée par l'ONG ou l'église aux centros de salud reliés au système de santé publique et à l'IESS (Institut équatorien de 
sécurité sociale): des milliers d'opérations, d'échanges et de compromis jalonnent le processus de consolidation des asentamientos.

6 Le phénomène de production de la ville s'accompagne de passages de formes de normativité très proches de l'habitat, du domestique, du familier, du sauvage à des normes et des équipements plus formalisés. Ce sont ces transformations qui préparent le passage au droit et à la ville normalisée. Des collectifs se constituent et se dissolvent. Par-delà des apparences trompeuses de l'anomie et de l'informel, les pratiques du droit et la mobilisation du travail juridique s'affirment, dans ce contexte particulier, à la fois comme des facteurs et des modalités de l'établissement des asentamientos dans les formes de la ville. Ce jeu de substitution de normativités n'aboutit pas nécessairement à davantage de sécurité.

7 Afin d'éclairer une telle ambivalence, prenons le parti méthodologique de recourir à une sélection d'images ${ }^{10}$. Limitons aussi le propos à la question de la propriété foncière et du logement. Les photographies introduites et commentées ci-dessous mettent en lumière une facette singulière d'un processus long et sinueux de constitution du foyer urbain. En bord de route, deux normativités caractéristiques d'un même dispositif de production de la propriété foncière se superposent et entrent en dialogue. Chacune se dispute le regard des usagers de l'avenue Casuarina, la principale artère permettant l'accès au secteur du Monte Sinaï.

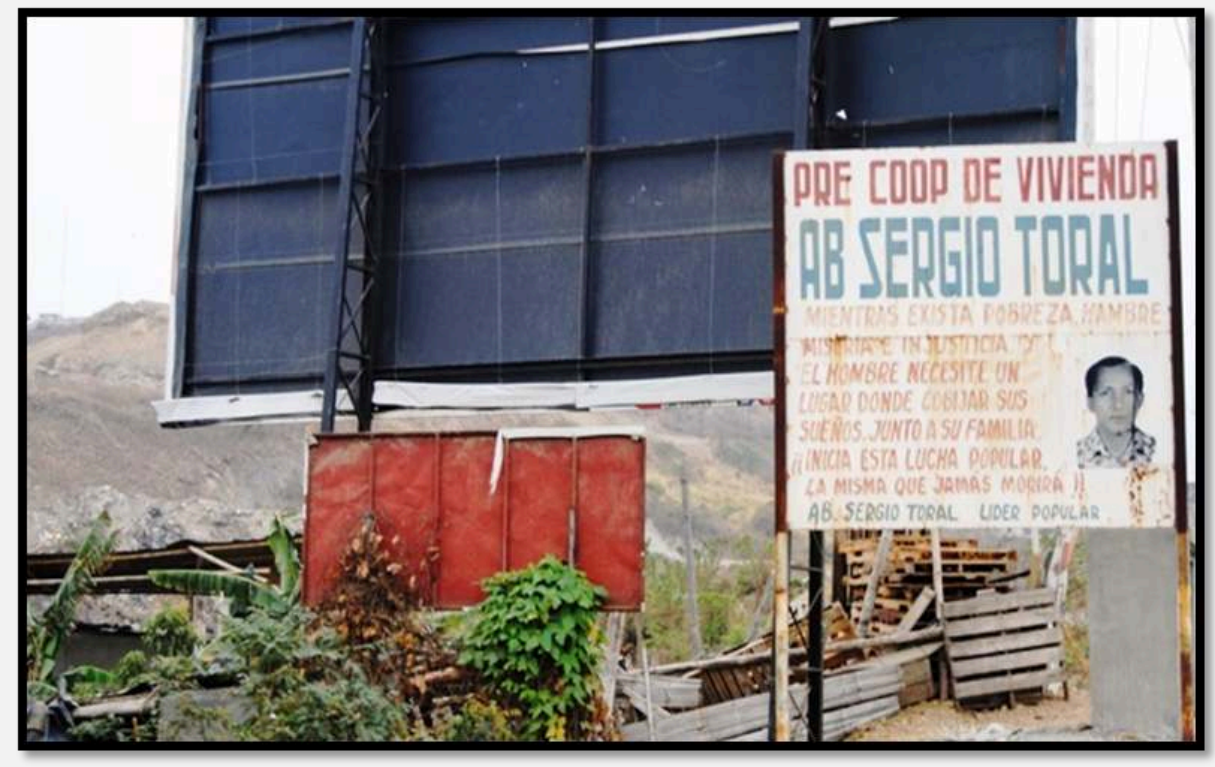




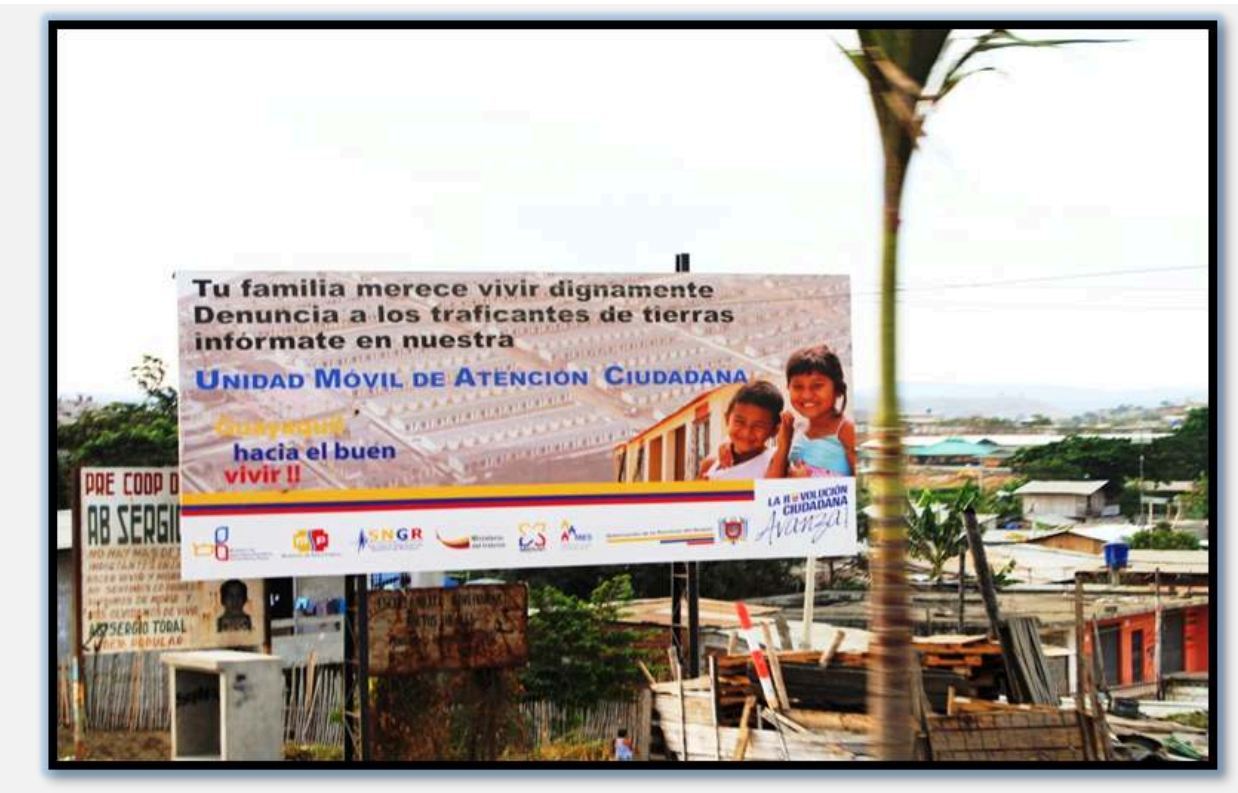

Considérons ces affiches dans leur rapport direct et non médiatisé aux usagers de l'avenue Casuarina.

\section{Affiche 1 : la formation de la « pré-coopérative » de logement Sergio Toral}

9 Le panneau figurant sur la première image, visiblement le plus ancien des deux, porte l'intitulé "Pré-coopérative de logement, avocat Sergio Toral ». L'affiche énonce ensuite sur un ton harangueur et sous la forme d'une assertion intemporelle :

« tant qu'existent la pauvreté, la faim, la misère, l'injustice et que l'homme a besoin d'un endroit où abriter ses rêves avec sa famille (cette lutte populaire commence et jamais ne mourra!). Avocat Sergio Toral, leader populaire».

10 L'usage du concept de "pré-coopérative", tout comme le référentiel convoqué invoquant la lutte populaire pour l'accès à un toit évoquent un premier mode historique d'accès au logement à travers l'asentamiento. Le panneau situe la formation de la pré-coopérative Sergio Toral au regard d'une série d'évènements inscrits dans la mémoire collective des habitants de Guayaquil. La naissance des premières coopératives coïncide en ce sens avec l'installation, au cours de la période cacaotière (fin XIX ${ }^{e}$-début $\left.\mathrm{XX}^{\mathrm{e}}\right)^{11}$, de campements de travailleurs temporaires et saisonniers. Les terres occupées, réputées sans maître, se sont concentrées, dans un premier temps, aux abords du port et du parc industriel en rapide expansion. Les premiers campements aujourd'hui pleinement assimilés à la trame urbaine se sont ensuite multipliés en zones inondables, à proximité des artères de communication principales, essentiellement sur des espaces considérés impropres à l'habitat ou au commerce. Gagnant du terrain sur la mangrove et le fleuve Guayas, les travaux de consolidation du sol se sont poursuivis par terrassement (« relleno», littéralement par «remplissage »). Les collines boisées d'un paysage, dont il ne reste désormais que certains îlots menacés, ont servi de matériel de remblais ${ }^{12}$. 
11 Le recul des écosystèmes et l'expansion de la ville par poches de peuplement acquiert une visibilité particulière durant la seconde moitié $\mathrm{du} \mathrm{XX}^{\mathrm{e}}$ siècle. Les enjeux sociopolitiques et spéculatifs associés au développement périurbain ont progressivement été mis au jour depuis les sphères de gouvernance locales et internationales alors que les concepts "d'invasions ", d'" asentamientos informales ", de "prises de terre collectives» gagnaient l'imaginaire collectif. Le patron de peuplement a pris ainsi progressivement, à partir des années 1950, les traits d'une occupation organisée, d'un mouvement. Cette dernière est systématiquement associée au démembrement d'anciennes exploitations agricoles, les haciendas, des « communes » rurales ou de propriétés publiques demeurées en friche.

À ces capacités d'organisation collective accrues demeure tout particulièrement associée l'émergence d'une figure publique locale: le «dirigeant» ou «leader populaire " auquel renvoie notre première affiche. Ce succès tient, de manière très générale, au développement d'un savoir-faire. Sur le plan logistique, ces acteurs locaux planifient et coordonnent l'occupation collective de larges surfaces en des temps restreints. Ils évaluent à travers un diagnostic préliminaire, qui est le propriétaire et, plus spécifiquement, l'imminence des droits susceptibles d'être revendiqués à l'encontre de l'entrée en possession. La pratique du droit dans le cadre de ce premier mode historique de territorialisation revêt une dimension à la fois revendicatrice et régulatrice.

Revendicatrice, en premier lieu, dès lors que le principe coutumier de fonction sociale de la propriété ${ }^{13}$ et le corpus des droits fondamentaux associés au logement se voient directement opposés aux propriétaires originaux (que ces derniers soient par ailleurs des personnes privées, publiques ou des communes rurales ancestrales). La création de la pré-coopérative répond, en ce sens, à la formation d'une association d'habitants dont l'objet serait de formaliser la revendication collective des droits d'acquisition, d'usage et de transformation du sol occupé à des fins d'habitation. La formation de la précoopérative signifie l'entrée en possession du groupe. Le panneau observé inscrit symboliquement la prétention collective des habitants dans l'espace public. Le préfixe " pré » implique, dans ce cas de figure, que la coopérative, personne morale porteuse de la revendication foncière, ne se trouve pas inscrite sur les registres officiels tenus par l'Etat ou la municipalité. La création de cette forme associative intermédiaire et, selon les points de vue, imparfaite, demeure néanmoins clairement identifiable et porteuse de sens depuis la perspective du droit positif équatorien. L'emprunt du format officiel de participation collective à la vie publique - la liberté d'association -, l'autocatégorisation comme "pré-coopérative de vivienda " ainsi que la dénomination des lieux-dits relèvent bien, dans ce cas de figure, d'une pratique de mise en forme même si elle n'aboutit pas forcément à celle du droit.

14 Régulatrice, en second lieu, dès lors que la mise en place d'une nouvelle forme de leadership répond historiquement à la formation et à la reconnaissance d'un ensemble de normes organisationnelles au sein de la collectivité. La production des normes de sécurisation de la possession, celles d'aménagement et de construction de la parcelle, de participation à la vie collective -la minga-, les normes de contribution financière aux dépenses communes, ponctuent les premiers temps de la territorialisation. Les solidarités et les liens de voisinage s'organisent en vue de consolider l'état de possession. La pratique du droit soulève, encore dans ce contexte, un enjeu plus classique de conseil et de représentation. Le travail du dirigeant tient, sur ce dernier 
plan, à l'exercice d'un pouvoir de médiation sociale. Ce dernier intervient auprès des acteurs gravitant en périphérie de l'asentamiento. Porte-voix des occupants, il négocie avec les autorités publiques et avec les propriétaires privés les conditions de régularisation du régime foncier à l'échelle de la coopérative. Ce dernier facilite accessoirement la mobilisation des votes en période électorale ${ }^{14}$, assurant un appui au candidat le plus offrant. Il sera le plus souvent rétribué en prenant lui-même possession d'un ou plusieurs terrains de la coopérative.

Une seconde interprétation possible du signal visuel analysé va généralement dans le sens, non seulement d'une évolution contemporaine, mais aussi d'une superposition des modes de formation des asentamientos à Guayaquil. Il s'agit, en d'autres termes, d'observer dans quelle mesure le même affichage peut, à la lumière d'un travail de contextualisation sociohistorique, faire l'objet d'une lecture sensiblement différente. La dualité et l'ambivalence attachées à la formation de la pré-coopérative Sergio Toral s'en trouvent ainsi progressivement relevées. La vague d'expansion urbaine la plus récente, entamée dans les années 1990, correspond aux premiers temps de formation des coopératives du Monte Sinaï. Cette période marque un net retrait de la figure charismatique du leader populaire. L'activisme des mouvements sociaux de gauche à l'origine des premières occupations collectives cède progressivement le pas au marché des parcelles habitables - prédios y solares -. La formation des nouveaux asentamientos répond plus nettement à une logique mercantile. Le peuplement s'opère, en somme, au gré d'une rencontre entre l'offre et la demande de logement à bas prix. Le patron de peuplement répond précisément au montage d'opérations de spéculation immobilière. La production de l'offre d'habitat urbain tend ainsi à se jouer dans des cercles de pouvoir "informés", extérieurs aux asentamientos déjà existants. Les trafics de terres anticipent l'action municipale ou étatique. Une nouvelle classe d'intermédiaires vend des paris sur l'avenir ${ }^{15}$. L'échange emprunte directement, une fois encore, aux formes classiques du transfert de propriété : le contrat de promesse de vente, la mise en location, le démembrement de la propriété et le transfert des droits d'usage. L'initiative de la vente provient parfois même des propriétaires terriens originaux. Ce type d'opération tend, tout du moins, à s'effectuer avec leur consentement et sous une forme négociée facilitant une prise de possession rapide et pacifique. Cette participation active des ayants droit originaux s'explique aisément dès lors que la valeur commerciale de la parcelle rurale démembrée, vendue comme habitable et, surtout, "habitée » par des familles augmente significativement. Le changement de destination de terrains ruraux - les "macro-solar »-, effectivement inscrits sur les registres de propriété publics, s'effectue alors en marge des cadres réglementaires. La vente de subdivisions constitue, cette fois, le cœur d'une irrégularité administrative. La problématique sociale de l'habitat apparait ici comme un moyen de capitalisation. L'offre irrégulière s'assure, de fait, un avantage compétitif face à tout type d'offre de logement concurrente. L'occupation matérielle et symbolique des lieux, fait, par effet d'inertie, levier sur l'action publique. Cette dernière n'affecte que partiellement la validité du transfert de propriété. Ricardo Pozo résume sur ce point :

“En Équateur, le terme d'invasions a traditionnellement été utilisé afin de désigner les établissements humains qui trouvaient leur origine dans une appropriation illégale ou prise de terre par des groupements de familles disposant de faibles revenus, sans le consentement de son propriétaire. Cependant, dans le présent cas d'étude, on assiste à une mécanique différente. (...) Les terrains ont été progressivement achetés légalement aux propriétaires originaux par divers promoteurs immobiliers informels ou trafiquants de terres. L'illégalité se situe au 
niveau de la parcellisation et de la vente de terrains ne se trouvant pas inscrits sur le registre de la propriété et qui n'appartiennent pas à des projets d'urbanisation antérieurement approuvés par l'administration publique locale. Cependant, dans la presse locale on continue à utiliser le terme de manière erronée ${ }^{16}$. " (Traduction personnelle)

Il semble intéressant d'observer, sur ce dernier point, que le dispositif d'affirmation de la possession tend, dans une large mesure, à s'effectuer avec le soutien de la société civile. La fondation jésuite Hogar de Cristo qui intervient sur la base de considérations humanitaires a historiquement fait figure d'acteur historique du processus de consolidation des asentamientos. L'organisation a développé une offre de solutions d'habitat temporaire préfabriquées et vendues à micro-crédit. La possession d'un écrit rédigé sous seing privé atteste l'achat du terrain et permet la délimitation physique des limites du solar. L'acte d'achat et l'installation de la maison préfabriquée à des fins de logement familial assoient la possession. L'un dans l'autre, ces éléments composites posent les bases d'une forme de revendication entendue par les autorités publiques. Le dispositif d'interpellation mis en place scelle l'entrée en lice des institutions publiques.

\section{Affiche 2 : le dispositif de régularisation ad hoc et l'office public de consolidation des droits}

17 La seconde image à l'étude renvoie précisément à l'intervention ad hoc des autorités publiques dans le contexte de consolidation de l'asentamiento. L'État produit rétroactivement une seconde normativité qui marque une nouvelle étape du processus de réalisation des droits. Le signal visuel à l'étude s'inscrit plus nettement cette fois dans une stratégie de communication et de reconquête de l'espace public:

"Ta famille mérite de vivre dans la dignité. Dénonce les trafiquants de terre. Information auprès de notre Unité Mobile d'attention citoyenne... Guayaquil en marche vers le Buen vivir... la révolution citoyenne avance!»

En trame de fond, le panneau suggère un mode d'urbanisation alternatif. La voie de consolidation insinuée est paradoxalement celle du plan de logement social plutôt que celle de la transformation du bâti existant. L'affichage sous-tend la possibilité pour les usagers de la Casuarina d'accéder à un habitat type, un standard vendu clé en main et à crédit. Ce type d'aménagement, schématisé en arrière-plan, rappelle l'offre des urbanisations et des condominiums privés. Ce mode d'accès à l'habitat est généralement réservé aux groupes de population bénéficiant des revenus les plus élevés. La proposition n'est plus endossée dans ce cas de figure par un acteur privé mais bien par un appareil d'État. Les logos institutionnels du ministère de l'Agriculture, de la Santé publique, de l'Intérieur, du Développement urbain et du Logement, de l'Inclusion économique et sociale et celui du Secrétariat de la gestion des risques apparaissent en ordre de bataille rangée. L'allusion au noyau familial et la symbolique du foyer sont, ici encore, sollicitées pour capter le regard des usagers de l'avenue Casuarina. La garantie $\mathrm{du}$ droit à une vie digne et le référentiel du «buen vivir» n'apparaissent plus, cependant, comme les contreparties d'efforts et de luttes collectives mais bien comme le produit d'un processus d'institutionnalisation et de formalisation du lien entre les citoyens et l'État. Ce dernier revendique à la fois une position de garant de l'ordre public, d'agent de la réalisation des droits et de fournisseur de services publics. L'inscription de ce signal dans le paysage urbain semble encore une fois susceptible 
d'une lecture plus nuancée et contextuelle. Il s'agit, en particulier, d'observer à travers l'évolution du cadre légal et réglementaire la versatilité des modes d'intervention publique participant à la territorialisation des asentamientos.

Ainsi, aux premiers jours de janvier 2010, afin de permettre la mise en place d'un Plan hydrique, le décret présidentiel $n^{\circ} 607$ déclarait « zone de sécurité » une surface de près de 9700 hectares à la périphérie nord-ouest de Guayaquil ${ }^{17}$. La mesure se traduit, en substance, par le tracé d'une zone tampon séparant d'un côté, un réseau de canaux artificiels destinés au captage des eaux pluviales et, de l'autre, des zones d'habitation non consolidées. Sous le sceau d'une mesure de sécurité publique ${ }^{18}$, l'espace en dispute est administrativement placé sous le contrôle des forces armées. Le motif est absent de la lettre du décret. Il se trouve néanmoins clairement exposé à travers les discours du président Correa ${ }^{19}$. Le gouvernement central souhaite mettre en effet un point d'arrêt à l'occupation foncière irrégulière dans le secteur nord-ouest de la ville. «Zéro tolérance aux invasions » : la sentence est prononcée. Les trafiquants de terre seront pénalement poursuivis. Une nouvelle autorité administrative est alors créée dans la continuité de l'annonce gouvernementale. La mission de ce «Comité interinstitutionnel de prévention des Établissements irréguliers ${ }^{20} »$ plus tard rebaptisée Secretaria Técnica de Prevención de Asentamientos irregulares (STAPHI) consiste à "proposer des politiques publiques pour prévenir, ordonner et contrôler les établissements humains irréguliers ainsi que coordonner et évaluer l'exécution interinstitutionnelle des politiques publiques mises en ceuvre dans le domaine $^{21} »$.

21 Près de 5000 familles des 28000 installées dans le secteur du Monte Sinaï sont directement concernées par l'exécution du décret $n^{\circ} 607$. L'État central propose aux foyers affectés, un plan de relogement dont la livraison des infrastructures est annoncée pour un futur proche : le plan de vivienda "Ciudad Victoria». Aux lendemains de la déclaration et de l'annonce d'une prochaine adjudication de parcelles aux familles établies à l'intérieur du périmètre de sécurité, près de 1125 familles incitées par la mesure de " compensation » sont venues édifier, en l'espace de quelques semaines, des structures de base dans cette même zone de sécurité. Les foyers qui nourrissaient clairement l'espoir d'intégrer le programme de relogement proposé aux futurs "déplacés " se trouvent désormais sous le coup de mesures d'éviction. Les principales voies d'accès au secteur sont placées sous surveillance policière. La vente de matériaux de construction est proscrite jusqu'à nouvel ordre. Au détour de cet état de siège, une solution de principe s'impose verticalement: annonce d'une offre publique de relogement et adjudication de lots pour les familles en situation d'irrégularité établies antérieurement à l'entrée en vigueur de la mesure (avant décembre 2010) ; éviction des familles «opportunistes » établies dans les semaines ayant suivi la publication du décret $n^{\circ} 607$ (après décembre 2010); poursuites pénales à l'encontre des trafiquants suspectés d'avoir réalisé des transactions illégales et contribué à la spéculation immobilière ${ }^{22}$.

Près de deux années après la parution du décret présidentiel $n^{\circ} 607$, une nouvelle intervention vient modifier pour partie le régime juridique de la zone de sécurité. Le motif officiel relève cette fois de la mise en œuvre d'une mesure de police environnementale et de la création d'une aire naturelle protégée (Déclaratoria de Bosque $y$ vegetacion protector del papagayo Guayaquil ${ }^{23}$ ). Au titre de ce reclassement en zone d'habitat naturel du perroquet de Guayaquil -une espèce locale protégée en voie d'extinction-, une large portion de la zone de sécurité passe sous la juridiction du 
ministère de l'Environnement ${ }^{24}$. À l'encontre de toute attente, l'initiative provient, non pas du ministère de l'Environnement, mais bien du ministère du Logement. La stratégie gouvernementale d'endiguement des asentamientos irréguliers en utilisant le motif écologique s'inscrit dans la continuité directe du décret $n^{\circ} 607$.

La parenthèse refermée, le régime juridique du secteur fait, l'année suivante, encore une fois l'objet d'une retouche d'ensemble. La réforme à la "Loi de légalisation de la possession foncière en faveur des habitants en possession de terrains localisés dans la circonscription territoriale des cantons de Guayaquil, Samborondón et El Triunfo ${ }^{25}$ » revient sur un conflit de juridiction ayant opposé la municipalité de Guayaquil au gouvernement central. Les coordonnées fixant les limites du secteur urbain sont réévaluées et étendues afin d'inclure les coopératives du Monte Sinaï. Les terrains relevant du patrimoine privé de l'État ou ayant fait l'objet d'une déclaration d'utilité publique en vue de leur expropriation demeureront administrés par le gouvernement central. La position de principe établie par le décret $n^{\circ} 607$ est donc reconduite et étendue par voie législative. Le Ministère du Développement urbain et de l'habitat (MIDUVI), secondé par le Comité de prévention des asentamientos irréguliers, prend en main la coordination des procédures de "légalisation " des titres de propriété. Près de 7000 certificats de possession géoréférencés, sur 16000 prévus au départ, sont remis aux familles dont l'installation, avant la date fatidique d'entrée en vigueur du décret décembre 2010 -, aura pu être attestée par relevé satellitaire ${ }^{26}$. Le reste du secteur est, quant à lui, placé sous la juridiction de la municipalité. Suite à ce remaniement, les habitants du secteur demeureront soumis à des agendas de régularisation concurrents. Alors que les vagues d'éviction se poursuivent, les questionnements restent en suspens quatre ans après l'entrée en lice du gouvernement central : quid de la légalité du décret présidentiel n 607 qui n'a jamais été mise en cause sur le fond ?; quid de l'égalité de traitement entre administrés si une seule et même population se trouve, suite aux ultimes soubresauts de la loi, sujette à deux juridictions, deux agendas de régularisation distincts et concurrents, l'un étant assumé par l'État central, l'autre par la municipalité de Guayaquil ${ }^{27}$ ?; quid des conditions procédurales d'exécution des mesures d'évictions, de l'expertise technique, de l'accès aux relevés satellitaires pris avant et après décembre 2010 et sur la base desquels les ordres d'éviction continuent d'être délivrés au jour le jour?; quid du caractère rétroactif et aléatoire de l'action publique intervenant à l'issue d'une période de laissez-faire de près d'une dizaine d'années, du principe de confiance légitime?; quid de l'exercice des droits de recours et de l'ouverture des voies de revendications juridictionnelles alors que le vécu du droit se limite encore pour beaucoup à celui d'une barrière linguistique et d'une situation attentatoire aux libertés fondamentales?; quid de la non-viabilité des mesures compensatoires, du plan de relogement 'Ciudad Victoria' et de l'accès à des solutions de logement 'alternatives' à travers l'offre publique de vivienda social ? Vers quelles entités les populations affectées vont-elles se tourner afin de se reloger : l'État, alors que la «livraison» des lotissements s'effectue au compte-goutte et, plus inquiétant sans doute, que le coût d'accès aux bénéfices du plan de relogement demeure inaccessible pour une large partie des foyers sous le coup d'une éviction prochaine? Ou le rôdeurvendeur de parcelles que les acheteurs savent entachées d'un avenir incertain mais qui demeurent, néanmoins, les seules accessibles économiquement?

24 A la lumière des éléments examinés, la quête d'un logement décent ne disparait pas avec la menace de l'éviction et plus généralement avec l'annonce d'une politique publique. Son flux ne fait, par définition, qu'être redirigé vers un prochain point de 
crispation du processus de territorialisation. Les trafiquants de terre se retirent progressivement du jeu alors que la présence de l'État s'intensifie.

De nouvelles vagues d'occupation reprennent dans d'autres secteurs périphériques de la ville. Par effet d'inertie, le principe de réalité prend progressivement le pas sur la situation de crise. L'attente, la résistance passive et les solidarités de voisinage $s^{\prime}$ 'instaurent comme des aléas du quotidien de territorialisation ${ }^{28}$. En dernière instance, la pression de l'institutionnalisation retombe. En pleine campagne électorale, le gouvernement central opère le tournant attendu. La troisième loi de réforme à la loi de régularisation du secteur proposé par le cabinet présidentiel et ce, près de sept années après la signature du décret de création de la zone de sécurité, fait sauter le verrou de l'illégalité29. La mesure, dont la justification repose cette fois explicitement sur la garantie des droits constitutionnels au logement, à une vie digne, sur celle du droit à la ville de même que sur la mise en œuvre du principe de non-discrimination ouvre la voie à une régularisation massive de la possession foncière dans le secteur du Monte Sinaï. Outre l'octroi de titres pour les propriétés utilisées à des fins d'habitation principale, le spectre de régularisation s'étend à la possession des immeubles utilisés à des fins de commerciales. En périphérie des asentamientos, l'Etat de droit moderne organise les conditions de sa propre subsistance. La reconnaissance ad hoc des droits acquis apparaît bel et bien, dans ce contexte, comme la fin d'un cycle. L'autorité publique est contrainte de replanter sa propre centralité ou, selon les points de vue, les conditions de sa légitimité comme forme régulatrice du processus urbain métropolitain.

\section{NOTES}

1. Voir DAVIS Mike, Planet of slums, Verso, New York, 2006; RAO Vyjayanthi, "Slum as theory: the South/Asian city and globalization", International Journal of Urban and Regional Research, vol. 30, no 1, 2006, pp. 225-232; GILBERT Alan, “The return of the slum: does language matter?", International Journal of Urban and Regional Research, vol., 31, n 4, p, 2007, 697-713; ARABINDOO, Pushpa. "Rhetoric of the 'slum' Rethinking urban poverty." City 15.6 (2011) : 636-646 ; SIMON, David. "Situating slums : Discourse, scale and place." City 15.6 (2011), pp. 674-685.

2. CASTEL Manuel, Crisis urbana, estado y movimientos sociales en las sociedades dependientes latinoamericanas, México, FCE, 1979, p. 1-2. David Harvey observe que « dès leurs origines les villes ont été édifiées à travers la concentration géographique et sociale du produit d'un surplus. L'urbanisation a toujours donc été liée à des phénomènes de classe considérant que ces surplus étaient extraits de quelque part ou de quelqu'un (en général une paysannerie oppressée) alors que le contrôle sur les dépenses de ce surplus repose typiquement dans un nombre limité de mains ", extrait de HARVEY David, "The right to the city", New Left review, volume 53, September-October 2008.

3. HART Keith, "Informal Income Opportunities and Urban Employment in Ghana", Journal of Modern African Studies, vol. II, 1972 ; ILO, "Employment, Incomes and Equality, A Strategy for Increasing Productive Employment in Kenya”, ILO, 1972, Geneva. 
4. ROY Ananya. "Urban informality : toward an epistemology of planning." Journal of the american planning association 71.2 (2005), pp. 147-158.

5. ESQUIROL Jorge, «Continuing Fictions of Latin American Law ", Florida Law review, volume 55, January 2003, p. 41; du meme auteur, "The Failed Law of Latin America", The American Journal of Comparative Law, volume 58, Winter 2008, p. 75.

6. UPHAM Frank, "Mythmaking in the Rule of Law orthodoxy", Rule of Law Series, Democracy and Rule of Law Project, Carnegie Working Papers, September 2002.

7. GARCÉS Chris, "Exclusión constitutiva: Las organizaciones pantalla y lo anti-social en la renovación urbana de Guayaquil", Iconos, Revista de Ciencias Sociales, 2004, no 20, p.53-63; ANDRADE, Xavier, “Mas ciudad, menos ciudadanía : renovación urbana y aniquilación del espacio público en Guayaquil”, Análisis, 2006

8. GOIRAND Camille, "'Philanthropes' en concurrence dans les favelas de Rio", Critique internationale, 1999, vol. 4, n 1, pp.155-167 ; ILLICH Ivan, "Las sombras de la caridad", Cidoc informa, vol. 4, $\mathrm{n}^{\circ}$ 3, febrero 1967

9. VARGAS LLOSA Mario, BATAILLON Gilles, "Rêve et réalité de l'Amérique latine ", Problèmes d'Amérique latine, 2010/3, n 77, pp. 9-23.

10. SILBEY Jessica, "Images in/of Law", NYL Sch. L. Rev. 57 (2012): 171; PORTER Elizabeth G., "Taking images seriously", Columbia Law Review (2014), pp. 1687-1782; JOHANSEN Steven J., ROBBINS Ruth Anne, “Art-iculating the Analysis: Systemizing the Decision to Use Visuals as Legal Reasoning”, 20 Legal Writing 57 (2015), 67 p.

11. PAZ Juan J., CEPEDA Miño, "La época cacaotera en Ecuador", en Sonia Fernández Rueda, Pontificia Universidad Católica del Ecuador, Facultad de Economía, Quito, marzo/abril de 2011, $\mathrm{n}^{\circ} 03$.

12. GODARD Henry, "Quito, Guayaquil : Evolución y consolidación en ocho barrios populares", Travaux de l'IFEA, Tomo n XLIV, revista Ciudad, Quito, 1988, p. 253 ; POZO Ricardo, "Crecimiento urbano informal en el noroeste de Guayaquil : de asentamientos piratas a zonas militarizadas", extrait de "Habitabilidad básica para todos", Auc revista de arquitectura, Facultad de arquitectura y diseño de la universidad católica de Santiago de Guayaquil, Enero 2012 ; TIEPOLO Maurizio, "the "barrio marginado regularización in Guayaquil", Centro città del terzo mondo, Politecnico di Torino, working paper $n^{\circ} 27,2007$.

13. ANKERSEN, Thomas T., RUPPERT Thomas, «Tierra y Libertad: the social function doctrine and land reform in Latin America", Tul. Envtl. LJ, 2006, vol. 19, p. 69.

14. GODART Henry, "Quito, Guayaquil : Evolución y consolidación en ocho barrios populares", Travaux de l'IFEA, Tomo n XLIV, Revista Ciudad, Quito, 1988, p. 253.

15. La vente de subdivisions, lots de 100 mètres carrés, "sans titre ", atteint un prix variant de 2000 à 12000 dollars selon la localisation du lot, le degré de consolidation du secteur et le verbe du marchand de rêves.

16. POZO Ricardo," Crecimiento urbano informal en el noroeste de Guayaquil : de asentamientos piratas a zonas militarizadas", extrait de "Habitabilidad básica para todos" Auc. revista de arquitectura, Facultad de arquitectura y diseño de la universidad católica de Santiago de Guayaquil, janvier 2012.

17. Registre officiel République d'Équateur (RORE) n 358 du 8 janvier 2010.

18. Le décret $n^{\circ} 607$ opère un renvoi au décret $n^{\circ} 433$ du 21 juin 2007, ROE $n^{\circ} 114$ du 27 juin 2007 qui prévoyait « la délimitation des espaces géographiques (...) dans le but de prévenir, minimiser, contrôler les risques, gérer et exécuter des mesures pour éviter l'aggravation de la situation géographique du secteur. (Traduction personnelle)»

19. Au cours de l'Enlace Ciudadano 197allocution hebdomadaire du président Rafael Correa-: "Este Gobierno, jamás va a apoyar una invasión, porque por pobre que seamos no podemos invadir una propiedad privada, segundo, con esa estrategia pierden los más pobres, porque es la ley de la selva y los pobres no son los más fuertes, son los más débiles", 20/11/2010. 
20. Comité Interinstitucional de Prevención de Asentamientos Irregulares. En pratique, l'action du Comité se limite essentiellement à la mise en œuvre d'une police des asentamientos à l'échelle nationale et à l'exécution d'une tâche de coordination interministérielle.

21. Article du décret dans sa version originale : «Artículo 1.- Créase el Comité Interinstitucional de Prevención de Asentamientos Humanos Irregulares, con la finalidad de proponer política pública para prevenir, ordenar y controlar los asentamientos humanos irregulares; coordinar la ejecución interinstitucional de dicha política; y evaluar sus resultados “ Decreto Ejecutivo 1227 del 28 de junio 2012, publicado en el Registro oficial número 747 del 17 de julio 2012.

22. Base légale : Código Penal (art. 575-A) : "Serán reprimidos con prisión de dos a cinco años los que con el propósito de sacar provecho personal y a título de dirigentes, organicen sus cooperativas e invadan tierras, tanto en la zona urbana como en la rural, atentando el derecho de propiedad privada".

23. Décret $n^{\circ} 105$ publié au registre officiel $n^{\circ} 791$ du mardi 18 septembre 2012

24. Superficie de 3602 sur les 9700 hectares au total.

25. "Ley Reformatoria a la Ley de Legalización de la Tenencia de Tierras a favor de los moradores y posesionarios de predios que se encuentran dentro de la circunscripción territorial de los cantones Guayaquil, Samborondón y El Triunfo" ; Registro Oficial N. 105, Lunes 21 de octubre de 2013.

26. 'Adjudicaciones se han entregado en Monte Sinai', el Telegrafo, samedi 22 février 2014.

27. MERA Gino, "Illegal settlements in Guayaquil, and the proposal to compete with them, Particular solution about progressive urbanization and low cost lots for the poorest of the poor", http://www.hdm.lth.se/fileadmin/hdm/alumni/papers/SDD_2009_242b/Gino_Mera_Giler_-

_Ecuador.pdf. (dernier accès juin 2017)

28. LEFEBVRE Henri, Critique de la vie quotidienne, Paris, L'Arche, 1981, vol. III ; « Quotidienneté », Encyclopcedia Universalis [en ligne]: «Dénominateur commun des activités, lieu et milieu des fonctionnalités, le quotidien peut aussi être analysé comme aspect uniforme des grands secteurs de la vie sociale : le travail, la famille et la vie privée, les loisirs. Ces secteurs, distincts comme formes, imposent à la pratique une structure, où se retrouve leur aspect commun : la passivité du spectateur devant des images, des paysages. Dans le travail, la passivité devant des décisions auxquelles le travailleur n'a aucune part. Dans la vie privée, la consommation imposée, puisque les choix sont dirigés et les besoins suscités par la publicité et les études de marché. Cette passivité générale est d'ailleurs inégalement répartie : elle pèse plus lourdement sur les femmes, sur la classe ouvrière, sur les employés qui ne sont pas des technocrates, sur la jeunesse, bref sur la majorité ; cependant, pas de la même façon, pas en même temps, pas sur tous à la fois ".

29. Voir "Ley reformatoria a Ley de Legalización de la Tenencia de Tierras a favor de los moradores y posesionarios de predios que se encuentran dentro de la circunscripción territorial de los cantones Guayaquil, Samborondón y El Triunfo" 8 de mayo 2017, RO 999.

\section{RÉSUMÉS}

Le travail présenté dévoile une modalité d'accès au logement dans les villes du Sud. Le commentaire de deux photographies saisissant un paysage quotidien de l'avenue Casuarina -la principale voie d'accès au secteur du Monte-Sinaï- située en périphérie de Guayaquil, sert de trame aux développements. Entreprise dans la continuité d'un terrain de recherche doctorale couvrant la période de 2010 à 2017 , la mise en dialogue de ces images contribue à saisir le passage 
de modes de normativité considérés, de prime abord, comme non juridiques, à une régulation du logement et de l'habitat empruntant aux formes juridiques modernes du droit 'de' ou 'à' la ville (la propriété foncière, le service public, la garantie de droits fondamentaux, etc.). Un éclairage original quant à la place du droit, considéré à la fois comme moyen et aléa déterminant la construction du rapport des habitants à l'espace urbain et la fondation des 'asentamientos', est offert.

The article unveils an access mechanism to housing in cities of the Global South. The commentary of two photos capturing a daily life landscape of the Casuarina Avenue -the main access road to the Monte Sinai sector- located on the outskirts of Guayaquil, is used as a frame for developments. Building upon a doctoral research fieldwork covering up the period from 2010 to 2017, visual contents' analysis contributes to seize a shift from normativity modes considered, at first, as non-legal, towards a social regulation of housing and urban habitat borrowing from modern forms of urban law and rights to the city (urban property, access to public services and infrastructures, state's guarantee of basic human rights, etc.). An original insight as to the place of law, both as a means and a hazard which shapes inhabitants' relationship to urban space and the foundation of human settlements, is provided.

La investigación expone una modalidad de acceso a la vivienda en ciudades del Sur. El comentario sobre dos fotografías que capturan un paisaje cotidiano de la Avenida Casuarina - la principal vía de acceso al sector del Monte Sinaí - en la periferia de Guayaquil, sirve como marco para el análisis. Enraizada en una investigación doctoral de campo abarcando el período de 2010 a 2017, la puesta en diálogo de éstas imágenes contribuye a visualizar el paso de modos de normatividad considerados a primera vista, como no jurídicos, hacia una regulación de la vivienda y del hábitat con base en las formas jurídicas modernas del derecho 'de' o 'a' la ciudad (propiedad urbana, servicios públicos, garantía de derechos humanos, etc.). El artículo ofrece una mirada original sobre el lugar del derecho entendido a la vez como un medio y una contingencia determinando la vinculación de los habitantes con el espacio urbano y la fundación de los 'asentamientos'.

INDEX

Mots-clés : villes du Sud, établissements humains informels, logement, géographies du droit, Guayaquil.

Palabras claves : ciudades del sur, establecimiento humano informal, vivienda, geografías del derecho, Guayaquil.

Keywords : cities of the global south, informal human settlements, housing, geographies of law, Guayaquil

\section{AUTEUR}

\section{MATHIAS PÉCOT}

Mathias PECOT est enseignant chercheur à l'Ecole supérieure polytechnique du Littoral, ESPOL (Faculté des sciences sociales et humaines, Campus Gustavo Galindo), Guayaquil, Equateur. 